\title{
Approximating Common Fixed Points of an Evolution Family on a Metric Space via Mann Iteration
}

\author{
Liang Luo, ${ }^{1,2}$ Rizwan Ullah, ${ }^{3}$ Gul Rahmat, ${ }^{3}$ Saad Ihsan Butt $\mathbb{D}^{4},{ }^{4}$ and Muhammad Numan ${ }^{5}$ \\ ${ }^{1}$ Key Laboratory of High Performance Ship Technology (Wuhan University of Technology), Ministry of Education, \\ Wuhan 430070, China \\ ${ }^{2}$ School of Transportation, Wuhan University of Technology, Wuhan 430070, China \\ ${ }^{3}$ Department of Mathematics, Islamia College Peshawar, Peshawar, KP, Pakistan \\ ${ }^{4}$ Department of Mathematics, COMSATS University Islamabad, Lahore Campus, Lahore, Pakistan \\ ${ }^{5}$ Department of Mathematics, COMSATS University Islamabad, Attock Campus, Attock, Pakistan \\ Correspondence should be addressed to Saad Ihsan Butt; saadihsanbutt@cuilahore.edu.pk
}

Received 4 March 2020; Revised 20 August 2020; Accepted 5 March 2021; Published 25 March 2021

Academic Editor: Ming-Sheng Liu

Copyright (c) 2021 Liang Luo et al. This is an open access article distributed under the Creative Commons Attribution License, which permits unrestricted use, distribution, and reproduction in any medium, provided the original work is properly cited.

In this article, we present the set of all common fixed points of a subfamily of an evolution family in terms of intersection of all common fixed points of only two operators from the family; that is, for subset $\mathfrak{M}$ of $\mathfrak{Q}$, we have $F(\mathfrak{M})=F\left(\mathfrak{Y}^{\left(\varrho_{1}, 0\right)}\right) \cap F\left(\mathfrak{Y}^{\left(\varrho_{2}, 0\right)}\right)$, where $\varrho_{1}$ and $\varrho_{2}$ are positive and $\left(\varrho_{1} / \varrho_{2}\right)$ is an irrational number. Furthermore, we approximate such common fixed points by using the modified Mann iteration process. In fact, we are generalizing the results from a semigroup of operators to evolution families of operators on a metric space.

\section{Introduction}

The theory of fixed points has a lot of applications in mathematics. The existence of a solution for a system is equivalent to the existence of a fixed point for a particular map associated with the system. Fixed point theory helps us for assurance of existence and uniqueness of solutions of differential equations, integral equations, algebraic system, and numerical equations. For further study of fixed point theory and applications, we refer to [1]. Since 1960, an extensive amount of work has been done in the field of fixed point theory. Belluce and Kirk [2,3], Browder and Bruck [4, 5], Lim [6], and DeMarr [7] have discussed fixed point for contractions and nonexpansive mappings on Banach spaces. However, in [8], the occurring of common fixed points of nonexpansive strongly continuous semigroups has been discussed via employing asymptotic type approach.

A family of bounded linear mappings $\widetilde{\mathfrak{S}}=\left\{S_{t}: t \geq 0\right\}$ is called a semigroup if it satisfies the following two conditions: $S_{0}=I$ and $S_{x+y}=S_{x} S_{y}$, for all $x$ and $y \geq 0$. Such families arise from the solution of the autonomous system:

$$
\begin{aligned}
& \mathfrak{J}(r)=\mathfrak{A}(r), \\
& \mathbb{J}(0)=\xi .
\end{aligned}
$$

The study of semigroups has a big role in the field of difference and differential equations. Although it is very important, still cannot be applied for the nonautonomous system:

$$
\begin{aligned}
& \dot{J}(r)=\mathfrak{A}(r) \rrbracket(r), \\
& \mathbb{J}(0)=\xi .
\end{aligned}
$$

For such a system, we need the idea of evolution family. A family $\mathfrak{L}=\left\{\mathfrak{Y}^{(r, l)} ; r \geq l \geq 0\right\}$ of bounded linear mappings on a metric space $\delta$ is said to be an evolution family if it satisfies the following two conditions:

(i) $\mathfrak{Y}^{(r, r)}=I$

(ii) $\mathfrak{Y}^{(r, s)} \mathfrak{Y}^{(s, l)}=\mathfrak{Y}^{(r, l)}$ for all $r \geq s \geq l \geq 0$

Such families are the generalized form of a semigroup.

Therefore, in our study, we investigate the common fixed points of an evolution family (instead of semigroup) for a 
hyperbolic metric space. For some recent published work on this topic, we refer to [9-11]. In fact, in [9], the following result is proved.

Theorem 1. Let $\mathfrak{M}=\left\{\mathfrak{Y}^{\left(\varrho_{1}, 0\right)}: \varrho_{1} \geq 0\right\}$ be a subfamily of an evolution family of nonexpansive and strongly continuous operators on a metric space $\sigma$. If $\varrho_{1}$ and $\varrho_{2}$ are two positive real numbers with restriction that $\left(\varrho_{1} / \varrho_{2}\right)$ is an irrational number, then

$$
\operatorname{AFPS}\left(\mathfrak{Y}^{\left(\varrho_{1}, 0\right)}\right) \cap \operatorname{AFPS}\left(\mathfrak{Y}^{\left(\varrho_{2}, 0\right)}\right)=\operatorname{AFPS}(\mathfrak{M})
$$

where AFPS mean approximate fixed point sequence of the operators.

In [9], authors discussed the approximate fixed point sequences, but this paper is about convergence to a common fixed point of an evolution family by using the Mann iteration process. Also, we present the set of all common fixed points by the intersection of all common fixed points of only two operators from the same family. Therefore, we mention that these are two different approaches for fixed points.

In [12], Khamsi and Buthinah discussed the fixed points of a semigroup on a metric space. Khamsi and Kozlowski $[13,14]$ presented fixed points for pointwise nonexpansive and pointwise contraction mapping in modular function spaces. Furthermore, the existence of fixed points for asymptotic and pointwise contractions and asymptotic and pointwise nonexpansive mappings has been discussed by Kirk and $\mathrm{Xu}$ in [15]. These results have been extended by Hussain and Khamsi [16] to metric spaces. In [17], Khamsi discussed the existence of nonlinear semigroups and gave some applications to differential equations in Musielak-Orlicz spaces.

\section{Definitions and Preliminary Results}

In this section, we present some definition which will be utilized in main results of this paper. Throughout the paper, we use the following notations for simplifications and to avoid repetitions:

$M_{1}: \mathbb{R}, \mathbb{R}^{+}, \mathbb{Q}^{c}, \mathbb{Q}$, and $\mathbb{Z}$ denotes the set of real, positive real, irrational, rational, and integer numbers, respectively

$M_{2}:(\sigma, d)$ denotes a metric space

$M_{3}:(\delta, d, H)$ denotes a hyperbolic metric space

$M_{4}: \varrho_{1}, \varrho_{2} \in \Delta \Longrightarrow \varrho_{1}, \varrho_{2} \in \mathbb{R}^{+}$with the restriction that $\left(\varrho_{1} / \varrho_{2}\right) \in \mathbb{Q}^{c}$

Definition 1 (see $[18]) .(\delta, d)$ is said to be convex metric space if for all $r, q$ in $\delta$, there exist a unique metric segment $[r, q] \in \mathfrak{F}$, and for any two points $p \in[r, q]$ and $\zeta \in[0,1]$, such that $p=(1-\zeta) r+\zeta q$, and the following holds:

$$
\begin{aligned}
& d(r, p)=\zeta d(r, q), \\
& d(p, q)=(1-\zeta) d(r, q),
\end{aligned}
$$

where $\mathfrak{F}$ is the set of all line segments between the points of \%.

Definition 2 (see [19]). A convex $(\widetilde{\delta}, d)$ is called hyperbolic metric space $(\delta, d, H)$ if for all $c, r, q$ in $\delta$ and $\zeta \in[0,1]$, and the following condition holds:

$$
d(\zeta c+(1-\zeta) r, \zeta c+(1-\zeta) q) \leq(1-\zeta) d(r, q) .
$$

A subset $D$ of $(\sigma, d, H)$ is convex if for all $r, q$ in $D$, and the line segment $[r, q]$ belongs to $D$.

Definition 3 (see [12]). A $(\delta, d, H)$ is uniformly convex if for all $\nu \in \delta$, and for all positive real numbers $\xi$ and $\varepsilon$, the following inequalities hold:

$$
\delta(\xi, \varepsilon)=\inf \left\{1-\frac{1}{\xi} d\left(\frac{1}{2} r+\frac{1}{2} q, v\right) ; d(r, v) \leq \xi, d(q, v) \leq \xi, d(r, q) \geq \xi \varepsilon\right\}>0
$$

The concept of convexity in a uniform sense was developed initially only for Banach spaces (see [20]), but latter on in [21], it was extended to metric spaces. For further details, see $[19,22,23]$.

Definition 4 (see [12]). A $(\widetilde{\delta}, d, H)$ is called strictly convex when we have

$$
d(\nu, r)=d(\nu, q)=d(\nu, \zeta r+(1-\zeta) q)
$$

for any $\nu, r, q \in \mho$ and $0<\zeta<1$, and then $r=q$.
Lemma 1 (see $[14,23])$. Let $(\widetilde{\delta}, d)$ be uniformly convex. Also, let $\left\{C_{n}\right\}$ in $\mho \sigma$ be a sequence of nonempty, nonincreasing, convex, closed, and bounded sets if $r \in \sigma$ and

$$
0<l=\lim _{n \longrightarrow \infty} d\left(r, C_{n}\right)<\infty .
$$

If $r_{n} \in C_{n}$ is with $d\left(r, r_{n}\right) \longrightarrow l$, then $\left\{r_{n}\right\}$ is a Cauchy sequence.

In $(\delta, d, H)$, if there exist any nonincreasing sequence of sets which are nonempty, convex, closed, bounded, and also their intersection is nonempty, such property is 
called the property $(P)$. Moreover $(\delta, d, H)$ is called complete if it has the property $(P)$. Similarly if $(\delta, d)$ is complete and uniformly convex, then it satisfies the property $(P)$; for more details, we refer to $[14,23,24]$.

Definition 5 (see [12]). Let $D$ is a nonempty subset of $(\delta, d)$ and $\mathfrak{S}=\left\{S_{x}, x \geq 0\right\}$ be a semigroup on $D$. The set of all common fixed points of the family $\mathbb{S}$ is define as

$$
F(\mathfrak{S})=\bigcap_{x \geq 0} F\left(S_{x}\right)
$$

Proposition 1 (see [12]). If $D$ is a nonempty and closed subset of $a(\tilde{\delta}, d)$, the family $\mathbb{S}=\left\{S_{x}: D \longrightarrow \Re ; x \geq 0\right\}$ be a semigroup of continuous operators. Moreover, if $\bar{C}=[0,+\infty)$, then

$$
F(\mathfrak{S})=\bigcap_{x \in C} F\left(S_{x}\right)
$$

Proposition 2 (see [12]). Let $D$ be a nonempty and closed subset of $a(\delta, d)$ and $\mathfrak{S}=\left\{S_{x}: D \longrightarrow \mathfrak{R}: x \geq 0\right\}$ be a semigroup on $D$. If $\bar{C}=[0,+\infty)$, then there exist $\varrho_{1}, \varrho_{2} \in \Delta$ as defined in $\left(M_{4}\right)$, such that

$$
\bigcap_{x \in C} F\left(S_{x}\right)=F\left(S_{\varrho_{1}}\right) \cap F\left(S_{\varrho_{2}}\right) .
$$

If we combine Propositions 1 and 2, we get following theorem.

Theorem 2 (see [12]). Let $D$ be a nonempty and closed subset of $a(\tilde{\delta}, d)$, and $\mathfrak{S}=\left\{S_{x}: D \longrightarrow \Re ; x \geq 0\right\}$ be a semigroup. Then, for any two $\varrho_{1}, \varrho_{2} \in \Delta$ as defined in $\left(M_{4}\right)$, we have

$$
F(\mathfrak{S})=\bigcap_{x \geq 0} F\left(S_{x}\right)=F\left(S_{\Theta_{1}}\right) \cap F\left(S_{\varrho_{2}}\right) .
$$

Definition 6. An operator $\Gamma: D \longrightarrow D$ is said to be nonexpansive if for any $r, q \in D$, the following inequality:

$$
d(\Gamma(r), \Gamma(q)) \leq d(r, q),
$$

holds. Moreover, $s$ is said to be fixed point of $\Gamma$ if for some $s \in D$ and $\Gamma(s)=s$. We denote by $F(\Gamma)$ which is the set of all fixed points of the operator $\Gamma$.

Definition 7. An evolution family $\mathfrak{Q}=\left\{\mathfrak{Y}^{(r, l)} ; r \geq l \geq 0\right\}$ of bounded linear operators on a $(\delta, d)$ is called nonexpansive, continuous, and periodic if the following holds:

(i) $d\left(\mathfrak{Y}^{(r, l)} u, \mathfrak{Y}^{(r, l)} v\right) \leq d(u, v), \quad \forall r \geq l \geq 0$, and $u, v \in$ o

(ii) $\lim _{r-l \longrightarrow 0} \mathfrak{Y}^{(r, l)} u=u, \forall u \in \sigma$

(iii) There exist $p \geq 0$ such that $\mathfrak{Y}^{(r+p, l+p)}=\mathfrak{Y}^{(r, l)}$ for all $r \geq l \geq 0$, respectively

The present paper deals with the study of exploring fixed points for a subfamily of evolution family. Let $\mathfrak{M}=\left\{\mathfrak{Y}^{(x, 0)} ; x \geq 0\right\} \quad$ be a subfamily of the family
$\mathfrak{L}=\left\{\mathfrak{Y}^{(r, s)} ; r \geq s \geq 0\right\}$ on a metric space $\widetilde{\delta}$, that is, $\varrho_{1}$ and $\varrho_{2}$ periodic, where $\varrho_{1}, \varrho_{2} \in \Delta$ as defined in $\left(M_{4}\right)$.

First, we define the set of all common fixed points of the above families $\mathfrak{Q}$ and $\mathfrak{M}$.

$$
\begin{gathered}
F(\mathfrak{Q})=\bigcap_{r \geq s \geq 0} F\left(\mathfrak{Y}^{(r, s)}\right), \\
F(\mathfrak{M})=\bigcap_{r \geq 0} F\left(\mathfrak{Y}^{(r, 0)}\right) .
\end{gathered}
$$

The following lemma will be useful in the proof of our main results.

Lemma 2 (see [5]). Let $D \subseteq \mho$ which is strictly convex $(\widetilde{\delta}, d, H)$. If $\Gamma_{1}, \Gamma_{1}: D \longrightarrow \widetilde{O}$ are nonexpansive operators such that $F\left(\Gamma_{1}\right) \cap F\left(\Gamma_{2}\right) \neq \varnothing$, then for each $0<\omega<1$ and for nonexpansive mapping $Y: D \longrightarrow \sigma$ such that $\Upsilon(s)=\omega \Gamma_{1}(s)+(1-\omega) \Gamma_{2}(s) \quad$ for $\quad s \in D$, we have $F(\Upsilon)=F\left(\Gamma_{1}\right) \cap F\left(\Gamma_{2}\right)$.

In the rest of the paper, we will denote the evolution family by $\mathfrak{Q}$, the subfamily of the evolution family by $\mathfrak{M}$, and the nonempty closed and convex subset of the metric space unless stated otherwise by $D$.

\section{Methodology}

In this paper, we provide results concerning the fixed points of a subfamily of a nonexpansive evolution family of operators. In fact, such results are presented in [12] for semigroups of nonexpansive bounded linear operators. So, in this paper, we use the methodology as used in [12]. Such results are new, and we do not find in the existing literature.

\section{Main Results}

In this section, we will present our main results concerning the fixed points of an evolution family of bounded linear operators in terms of intersection of sets of fixed points of two operators from the family.

Proposition 3. Let $\mathfrak{Q}=\left\{\mathfrak{Y}^{(r, l)} ; r \geq l \geq 0\right\}$ be an evolution family on D. If $C \subset[0,+\infty)$ such that $\vec{C}=[0,+\infty)$, then

$$
F(\mathfrak{Q})=\bigcap_{r \geq l \geq 0} F\left(\mathfrak{Y}^{(r, l)}\right)=\bigcap_{c \geq d \geq 0 \in C} F\left(\mathfrak{Y}^{(c, d)}\right) .
$$

Proof. Clearly $F(\mathfrak{L}) \subset \cap \cap_{(c, d) \in C} F\left(\mathfrak{Y}^{(c, d)}\right)$. We only need to prove that

$$
\left.\bigcap_{(c, d) \in C} F\left(\mathfrak{Y}^{(c, d)}\right) \subset F(\mathfrak{Y})\right) .
$$

Let $s \in \cap_{(c, d) \in C} F\left(\mathfrak{Y}^{(c, d)}\right)$ which means $\cup(c, d) s=s$ for all $c \geq d \geq 0$, and $c, d \in D$. We need to show that $\mathfrak{Y}^{(r, l)} s=s$ for all $r \geq l \geq 0$. As $\bar{C}=[0,+\infty)$, then by denseness property, there exist sequences $c_{n}, d_{n} \in C$ such that $c_{n} \longrightarrow x$ and $d_{n} \longrightarrow y$ and $c_{n} \geq d_{n}$. Now, consider $\mathfrak{Y}^{\left(c_{n}, d_{n}\right)^{n}} s=s$ for all $s \in \mathfrak{R}$.

Now taking limit on both sides, that is, $\lim _{n \longrightarrow \infty} \mathfrak{Y}^{\left(c_{n}, d_{n}\right)} s=\lim _{n \longrightarrow \infty} s$ for all $s \in \mathfrak{R}$ gives 
$\mathfrak{Y}^{(r, l)} s=s$ for all $r \geq l \geq 0 . \quad$ Hence, $\quad s \in F(\mathfrak{L})$, $\cap_{(c, d) \in C} F\left(\mathfrak{Y}^{(c, d)}\right) \subset F(\mathfrak{Q})$. We get

$$
F(\mathfrak{Q})=\bigcap_{r \geq l \geq 0} F\left(\mathfrak{Y}^{(r, l)}\right)=\bigcap_{(c, d) \in C} F\left(\mathfrak{Y}^{(c, d)}\right) .
$$

Proposition 4 (see [25]). If $C \subset \mathbb{R}$ is nonempty and additive subgroup, then either $\bar{C}=\mathbb{R}$ or for nonnegative number $k$, we have $C=k \cdot \mathbb{Z}=\{k n, n \in \mathbb{Z}\}$. If there exist two real numbers $\varrho_{1}, \varrho_{2} \in \Delta$ as defined in $\left(M_{4}\right)$, then the set

$$
C\left(\varrho_{1}, \varrho_{2}\right)=\left\{n \varrho_{1}+m \varrho_{2} ; n, m \in \mathbb{Z}\right\},
$$

is dense in $\mathbb{R}$. Moreover, if $W=C\left(\varrho_{1}, \varrho_{2}\right) \cap[0,+\infty)$, then $\bar{W}=[0,+\infty)(W$ is dense in $[0,+\infty))$.

Proposition 5. Let $\mathfrak{M}=\left\{\mathfrak{Y}^{(r, 0)}: r \geq 0\right\}$ be subfamily of evolution family from $D$ into $\mho$. If $\varrho_{1}, \varrho_{2} \in \mathbb{R}^{+}$such that these numbers are also the periods of the evolution family, then

$$
\bigcap_{r \in C} F\left(\mathfrak{Y}^{(r, 0)}\right)=F\left(\mathfrak{Y}^{\left(\varrho_{1}, 0\right)}\right) \cap F\left(\mathfrak{V}^{\left(\varrho_{2}, 0\right)}\right),
$$

where $C=\left\{n \varrho_{1}+m \varrho_{2} ; n, m \in \mathbb{Z}\right\} \cap[0,+\infty)$.

Proof. As $\varrho_{1}$ and $\varrho_{2}$ are in $C$, therefore $\cap_{r \in C} F\left(\mathfrak{Y}^{(r, 0)}\right) \subset F\left(\mathfrak{Y}^{\left(\varrho_{1}, 0\right)}\right) \cap F\left(\mathfrak{Y}^{\left(\varrho_{2}, 0\right)}\right)$. We need only to prove that

$$
F\left(\mathfrak{Y}^{\left(\varrho_{1}, 0\right)}\right) \cap F\left(\mathfrak{Y}^{\left(\varrho_{2}, 0\right)}\right) \subset \bigcap_{r \in C} F\left(\mathfrak{Y}^{(r, 0)}\right) .
$$

Let $s \in F\left(\mathfrak{Y}^{\left(\varrho_{1}, 0\right)}\right) \cap F\left(\mathfrak{Y}^{\left(\varrho_{2}, 0\right)}\right)$, then $\mathfrak{Y}^{\left(\varrho_{1}, 0\right)} s=s$ and $\mathfrak{Y}^{\left(\varrho_{2}, 0\right)} s=s$. Let $r \in D$, then $r=n \varrho_{1}+m \varrho_{2}$.

Consider

$$
\begin{aligned}
& \mathfrak{Y}^{(r, 0)} \boldsymbol{s}=\mathfrak{Y}^{\left(n \varrho_{1}+m \varrho_{2}, 0\right)} \boldsymbol{s}=\mathfrak{Y}^{\left(n \varrho_{1}, 0\right)} \mathfrak{Y}^{\left(m \varrho_{2}, 0\right)} \\
& =\mathfrak{Y}^{\mathfrak{n}\left(\varrho_{1}, 0\right)} \mathfrak{Y}^{\mathfrak{n}\left(\varrho_{2}, 0\right)} s=\mathfrak{Y}^{\mathfrak{n}\left(\varrho_{1}, 0\right)} s=s .
\end{aligned}
$$

Hence, $F\left(\mathfrak{Y}^{\left(\varrho_{1}, 0\right)}\right) \cap F\left(\mathfrak{Y}^{\left(\varrho_{2}, 0\right)}\right) \subset \cap_{r \in C} F\left(\mathfrak{Y}^{(r, 0)}\right)$, where we use the property $U(0,0)$ which is the identity-map and consider $r=n \varrho_{1}-m \varrho_{2}$, where either $n$ or $m$ is negative. Assume that $r+m \varrho_{2}=n \varrho_{1}$, then

$$
\begin{aligned}
\mathfrak{Y}^{(r, 0)} \boldsymbol{s} & =\mathfrak{V}^{(r, 0)} \mathfrak{V}^{\mathfrak{m}\left(\varrho_{2}, 0\right)} \boldsymbol{s}=\mathfrak{V}^{(r, 0)} \mathfrak{Y}^{\left(m \varrho_{2}, 0\right)} \boldsymbol{s} \\
& =\mathfrak{Y}^{\left(r+m \varrho_{2}, 0\right)} \boldsymbol{s}=\mathfrak{Y}^{\left(n \varrho_{1}, 0\right)} \boldsymbol{s}=\mathfrak{V}^{\mathfrak{n}\left(\varrho_{1}, 0\right)} \boldsymbol{s}=\boldsymbol{s} .
\end{aligned}
$$

Hence, $s \in F\left(\mathfrak{Y}^{(r, 0)}\right)$ for any $r \in C$, and this completes the proof.

Theorem 3 (see [26]). Let $\mathfrak{M}=\left\{\mathfrak{Y}^{(r, 0)}: r \geq 0\right\}$ be subfamily of the evolution family on $D$. If $\varrho_{1}, \varrho_{2} \in \Delta$ as defined in $\left(M_{4}\right)$, then

$$
F(\mathfrak{M})=F\left(\mathfrak{Y}^{\left(\varrho_{1}, 0\right)}\right) \cap F\left(\mathfrak{Y}^{\left(\varrho_{2}, 0\right)}\right) .
$$

In particular, we have

$$
F(\mathfrak{M})=F\left(\mathfrak{Y}^{(2,0)}\right) \cap F\left(\mathfrak{Y}^{(\sqrt{3}, 0)}\right)=F\left(\mathfrak{Y}^{(\pi, 0)}\right) \cap F\left(\mathfrak{Y}^{(e, 0)}\right) .
$$

Theorem 4. Let $\mathfrak{M}=\left\{\mathfrak{Y}^{(r, 0)}: r \geq 0\right\}$ be subfamily of evolution family from $D$ into $\%$ which is continuous and nonexpansive. If $\varrho_{1}, \varrho_{2} \in \Delta$ as defined in $\left(M_{4}\right)$, then

$$
F(\mathfrak{M})=F\left(\zeta \mathfrak{Y}^{\left(\varrho_{1}, 0\right)}+(1-\zeta) \mathfrak{Y}^{\left(\varrho_{2}, 0\right)}\right),
$$

where $\zeta \in(0,1)$.

Proof. Let us consider $r \in F(\mathfrak{M})=F\left(\mathfrak{Y}^{\left(\varrho_{1}, 0\right)}\right) \cap F\left(\mathfrak{Y}^{\left(\varrho_{2}, 0\right)}\right)$. This ensures that $\mathfrak{Y}^{\left(\varrho_{1}, 0\right)} r=r$ and $\mathfrak{Y}^{\left(\varrho_{2}, 0\right)} r=r$. Now,

$$
\begin{aligned}
\left(\zeta \mathfrak{V}^{\left(\varrho_{1}, 0\right)}+(1-\zeta) \mathfrak{Y}^{\left(\varrho_{2}, 0\right)}\right) r & =\zeta \mathfrak{Y}^{\left(\varrho_{1}, 0\right)} r+(1-\zeta) \mathfrak{V}^{\left(\varrho_{2}, 0\right)} r \\
& =\zeta r+(1-\zeta) r=r .
\end{aligned}
$$

Hence, $r \in F\left(\zeta \mathfrak{Y}^{\left(\varrho_{1}, 0\right)}+(1-\zeta) \mathfrak{Y}^{\left(\varrho_{2}, 0\right)}\right)$.

Again consider $r \in F\left(\zeta \mathfrak{Y}^{\left(\varrho_{1}, 0\right)}+(1-\zeta) \mathfrak{Y}^{\left(\varrho_{2}, 0\right)}\right)$ which implies $\left(\zeta \mathfrak{Y}^{\left(\varrho_{1}, 0\right)}+(1-\zeta) \mathfrak{Y}^{\left(\varrho_{2}, 0\right)}\right) r=r$ or $\zeta \mathfrak{Y}^{\left(\varrho_{1}, 0\right)} r+(1-\zeta)$ $\mathfrak{V}^{\left(\varrho_{2}, 0\right)} r=r$.

If we take $\lim _{\zeta \longrightarrow 0}$ and $\lim _{\zeta \longrightarrow 1}$, it gives $\mathfrak{Y}^{\left(\varrho_{2}, 0\right)} r=r$ and $\mathfrak{Y}^{\left(\varrho_{1}, 0\right)} r=r$, respectively. Hence, $r \in F\left(\mathfrak{Y}^{\left(\varrho_{1}, 0\right)}\right) \cap F\left(\mathfrak{Y}^{\left(\varrho_{2}, 0\right)}\right)$ $=F(\mathfrak{M})$. This completes the proof.

\section{Approximating of Fixed Points of an Evolution Family}

In this section, we discuss the behavior of Mann iteration process by two operators, using previous results. With the support of these iterations, we will approximate the common fixed points of subfamily of an evolution family.

Definition 8. Let $T: D \longrightarrow D$, which is nonexpansive mapping and $0<\zeta<1$. Also let $M(T, \zeta)$ represent the Mann iteration process produced by the constant $\zeta$ and the mapping $T$, which is given by following iteration formula:

$$
s_{n+1}=\zeta T s_{n}+(1-\zeta) s_{n}
$$

where $s_{1}$ is chosen arbitrarily in $D$.

Lemma 3 (see $[14,23])$. Let $(\delta, d, H)$ is uniformly convex metric space and $v \in[0,+\infty)$ such that

$$
\begin{aligned}
& \limsup _{n \longrightarrow \infty} d\left(s_{n}, h\right) \leq v, \\
& \limsup _{n \longrightarrow \infty} d\left(t_{n}, h\right) \leq v, \\
& \lim _{n \longrightarrow \infty} d\left(h, \zeta s_{n}+(1-\zeta) t_{n}\right)=v,
\end{aligned}
$$


for some $0<\zeta<1$. Then,

$$
\lim _{n \longrightarrow \infty} d\left(s_{n}, t_{n}\right)=0 .
$$

Lemma 4. Let $(\delta, d, H)$ is uniformly convex and $T$ be a nonexpansive mapping defined from $D$ to $D$. Let $0<\zeta<1$ and $s_{n}$ be a sequence defined by (27). Suppose $\omega \in F(T)$. Then, $\lim _{n \rightarrow \infty} d\left(s_{n}, \omega\right)$ exists.

Proof. Let us consider for all $n \geq 1$,

$$
\begin{aligned}
d\left(s_{n+1}, \omega\right) & =d\left(\zeta T s_{n}+(1-\zeta) s_{n}, \omega\right) \\
& \leq \zeta d\left(T s_{n}, \omega\right)+(1-\zeta) d\left(s_{n}, \omega\right) \\
& =\zeta d\left(T s_{n}, T \omega\right)+\left(1-\zeta d\left(s_{n}, \omega\right)\right) \\
& \leq \zeta d\left(s_{n}, \omega\right)+(1-\zeta) d\left(s_{n}, \omega\right) \\
& =d\left(s_{n}, \omega\right) .
\end{aligned}
$$

Since the sequence $\left\{d\left(s_{n}, \omega\right)\right\} \geq 0$ and is nonincreasing, it is bounded below, and hence it is convergent. Next, we define asymptotic radius of a sequence.

Definition 9 (see [12]). Let $D$ be a nonempty subset $(\delta, d)$ and $\left\{s_{n}\right\}$ be a bounded sequence in $\sigma$. Define $r\left(.,\left\{s_{n}\right\}\right): D \longrightarrow[0, \infty)$ by

$$
r\left(s,\left\{s_{n}\right\}\right)=\limsup _{n \longrightarrow \infty} d\left(s, s_{n}\right) .
$$

The asymptotic radius of the sequence $\left\{s_{n}\right\}$ with respect to $D$ is denoted by $\rho_{D}$ and is defined by the following:

$$
\rho_{D}=\inf \left\{r\left(s,\left\{s_{n}\right\}\right): s \in D\right\},
$$

where $\rho$ is used to represent the asymptotic radius of the sequence $\left\{s_{n}\right\}$ with respect to $\delta$. Let $\xi \in D$ such that

$$
r\left(\xi,\left\{s_{n}\right\}\right)=r\left(D,\left\{x_{n}\right\}\right)=\min \left\{r\left(s,\left\{s_{n}\right\}\right): s \in D\right\} .
$$

Then, $\xi$ is an asymptotic center of sequence $\left\{s_{n}\right\}$ with respect to $D$.

Let $A\left(D,\left\{s_{n}\right\}\right)$ represent the collection of all asymptotic centers of $\left\{s_{n}\right\}$ with respect to $D$. When $D=\delta$, we use the notation $A\left(\left\{s_{n}\right\}\right)$. Generally, the set $A\left(D,\left\{s_{n}\right\}\right)$ of a bounded sequence $\left\{s_{n}\right\}$ contains more than one point. It can be empty too; for more details, we refer to [23].

Kuczumow [27] and Lim [6] offered an approach to weak convergence, which they called $\Delta$-convergence. In the case of CAT (0) spaces, this approach was very effective and successful.

Definition 10. Let CAT $(0)$ be a bounded sequence in $(\widetilde{\delta}, d)$. This sequence is called $\Delta$-converge to $s \in \sigma$ if and only if for every subsequence $\left\{u_{n}\right\}$ of $\left\{s_{n}\right\}, s$ is the unique asymptotic center of this subsequence. When $\left\{s_{n}\right\}$ is $\Delta$-converge to $s$, we denote by $s_{n} \longrightarrow^{\Delta} s$.

Next, we discuss the iteration scheme (27) for nonexpansive mappings, and particularly, we will investigate the $\Delta$-convergence of hyperbolic spaces which are uniformly convex.
Lemma 5 (see $[23,28])$. If $(\delta, d, H)$ is uniformly convex, then with respect to $D$, there exist a unique asymptotic center for each bounded sequence $\left\{s_{n}\right\} \in \widetilde{O}$.

Lemma 6 (see $[23,28])$. If $(\delta, d, H)$ is uniformly convex and $\left\{s_{n}\right\} \in D$ is a bounded sequence such that $A\left(\left\{s_{n}\right\}\right)=\{t\}$ and $r\left(\left\{s_{n}\right\}\right)=\rho$ and let $\left\{t_{m}\right\} \in D$ is a sequence such that $\lim _{m \longrightarrow \infty} r\left(t_{m},\left\{s_{n}\right\}\right)=\rho$, then $\lim _{m \longrightarrow \infty} t_{m}=t$.

In uniformly convex Banach spaces, D. Gohde gave the demi-closed principle [29]; a similar concept is discussed in next theorem.

Lemma 7. Let $(\widetilde{\delta}, d, H)$ be complete and uniformly convex and $T: D \longrightarrow D$ is a nonexpansive mapping on $D$. If $\left\{s_{n}\right\} \in D$ be a sequence such that $\lim _{n \rightarrow \infty} d\left(s_{n},\left\{T s_{n}\right\}\right)=0$ and if $s \in D$ is the asymptotic center of the sequence $\left\{s_{n}\right\}$ with respect to $D$, then $s \in T$, in certain, if $T$ has approximate fixed point sequence $\left\{s_{n}\right\} \in D$, such that $s_{n} \longrightarrow^{\Delta}$ s, then $s \in F(T)$.

In order to study the iterative behavior defined by (27), we give the following necessary theorem.

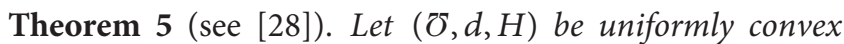
and $T$ be as defined in Lemma 7 , which has a fixed point. If $0<\zeta<1, s_{1} \in D$, and $\left\{s_{n}\right\}$ is generated by (27), then

$$
\lim _{n \longrightarrow \infty} d\left(T s_{n}, s_{n}\right)=0,
$$

and $T$ has an approximate fixed point sequence $\left\{s_{n}\right\}$.

The condition that $T$ has a fixed point that can be removed if we assume that $D$ is bounded (see [23]). Now, we study the $\Delta$-convergence of the Mann iteration process given in (27).

Theorem 6. Let $(\delta, d, H)$ be uniformly convex and $T$ be as defined in Lemma 7, which has a fixed point. If $0<\zeta<1$, $s_{1} \in D$, and $\left\{s_{n}\right\}$ is generated by (27), then $\left\{s_{n}\right\}$ is $\Delta$-converge to $s \in F(T)$.

Proof. The fixed set of operator $T$ is convex and closed, as given in [23]. Applying Theorem 5, $T$ has approximate fixed point sequence $\left\{s_{n}\right\}$, which implies $\lim _{n \longrightarrow \infty} d\left(T s_{n}, s_{n}\right)=0$, if the sequence $\left\{s_{n}\right\}$ has a unique asymptotic center $s$. Then, using Lemma 7, $s \in F(T)$. Now, we prove that $\left\{s_{n}\right\}$ is $\Delta$-converge to $s$. Suppose that the sequence $\left\{s_{n}\right\}$ has a subsequence $\left\{s_{n_{i}}\right\}$ and suppose this subsequence has the unique asymptotic center $z$. Again since $T$ has an approximate fixed point sequence $\left\{s_{n_{i}}\right\}$, we conclude that $z \in F(T)$.

Hence,

$$
\limsup _{n_{i} \longrightarrow \infty} d\left(s_{n_{i}}, z\right) \leq \limsup _{n_{i} \longrightarrow \infty} d\left(s_{n_{i}}, s\right) .
$$

Since $s, z \in F(T)$, we get

$$
\begin{aligned}
& \limsup _{n_{i} \longrightarrow \infty} d\left(s_{n_{i}}, z\right)=\lim _{n \longrightarrow \infty} d\left(s_{n}, z\right), \\
& \limsup _{n_{i} \longrightarrow \infty} d\left(s_{n_{i}}, s\right)=\lim _{n \longrightarrow \infty} d\left(s_{n}, s\right) .
\end{aligned}
$$


Since $\left\{s_{n_{i}}\right\}$ has the unique asymptotic center $s, s=z$. This shows that $\left\{s_{n}\right\} \Delta$-converge to $s$.

The combination of Theorems 3 and 6 gives the result, which is discussed in the next theorem.

Theorem 7. Let $(\sigma, d, H)$ is complete and uniformly convex with $D$ be a subset of $\delta$. If $\mathfrak{M}=\left\{\mathfrak{Y}^{(r, 0)}: r \geq 0\right\}$ is a subfamily of an evolution family on $D$ which is continuous and nonexpansive, if $\varrho_{1}, \varrho_{2} \in \Delta$ as defined in $\left(M_{4}\right)$ and $0<\alpha, \beta<1$ are such that $\alpha+\beta \leq 1$, and also if $s \in D$ and $\left\{s_{n}\right\} \in D$ be a sequence defined by

$$
s_{n+1}=(\alpha+\beta)\left(\alpha \mathfrak{Y}^{\left(\varrho_{1}, 0\right)}\left(s_{n}\right)+\beta \mathfrak{Y}^{\left(\varrho_{2}, 0\right)}\left(s_{n}\right)\right)+(1-\alpha-\beta) s_{n},
$$

where $n \geq 1$, then the sequence $\left\{s_{n}\right\}$ is $\Delta$-converge to a common fixed point of $\mathfrak{M}=\left\{\mathfrak{Y}^{(r, 0)} ; r \geq 0\right\}$.

Proof. Set

$$
S=\frac{\alpha}{\alpha+\beta} \mathfrak{Y}^{\left(\varrho_{1}, 0\right)}+\frac{\beta}{\alpha+\beta} \mathfrak{Y}^{\left(\varrho_{2}, 0\right)} .
$$

Observe that $S: D \longrightarrow D$ is nonexpansive. Clearly we have $F(\mathfrak{M}) \subset F(S)$. Let $s_{1} \in D$ and $\left\{s_{n}\right\}$ is the sequence produced by (37). Then,

$$
s_{n+1}=\zeta S\left(s_{n}\right)+(1-\zeta) s_{n},
$$

where $\zeta=\alpha+\beta \in(0,1)$. It means that the sequence $\left\{s_{n}\right\}$ is the same as produced by (27) for the operator $S$. According to Theorem $6,\left\{s_{n}\right\} \Delta$-converge to some $s \in F(S)$. By Theorem 3 , we know that $F(\mathfrak{M})=F(S)$; hence, $s \in F(\mathfrak{M})$ which completes the proof.

We conclude this section by the following important remark.

Remark 1. If we put the condition of periodicity on the family $\mathfrak{Q}$ for every positive real number, then it becomes a semigroup. So, in this case, Propositions 3.1, 3.2, and Theorems 3.2 and 4.3 in [12] become subcases of our work.

\section{Conclusion}

In this paper, we established the results concerning the fixed points of a subfamily of an evolution family on a metric space. It is more difficult to derive or find the set of all common fixed points for a family than to obtain the set of all common fixed points only for two maps. So, in this paper, we provide results which make it easy to present such set of fixed points in terms of only two maps from the same family. Also, we approximated the common fixed points for subfamily of evolution family using the well-known Mann iteration process. In future, we are trying to generalize these results for periodic evolution families which seem to be more applicable and fruitful for researchers working in this field or others related to fixed point theory. Recently, we completed more projects in this area, and we hope to be publish them in near future (see [30-32]).

\section{Data Availability}

The data used to support the findings of this study are included within the article.

\section{Conflicts of Interest}

The authors declare that they have no conflicts of interest.

\section{Authors' Contributions}

All authors contributed equally to this paper.

\section{Acknowledgments}

This research was supported by the Natural Science Fund of Anhui Wenda University of Information Engineering (grant no. XZR2019A10).

\section{References}

[1] W. A. Kirk and B. Sims, Handbook of Metric Fixed Point Theory, Springer Science \& Business Media, Berlin, Germany, 2001.

[2] L. Belluce and W. Kirk, "Fixed-point theorems for families of contraction mappings," Pacific Journal of Mathematics, vol. 18, no. 2, pp. 213-217, 1966.

[3] L. P. Belluce and W. A. Kirk, "Nonexpansive mappings and fixed-points in Banach spaces," Illinois Journal of Mathematics, vol. 11, no. 3, pp. 474-479, 1967.

[4] F. E. Browder, "Nonexpansive nonlinear operators in a Banach space," Proceedings of the National Academy of Sciences, vol. 54, no. 4, pp. 1041-1044, 1965.

[5] R. E. Bruck, "Properties of fixed-point sets of nonexpansive mappings in Banach spaces," Transactions of the American Mathematical Society, vol. 179, p. 251, 1973.

[6] T. C. Lim, "A fixed point theorem for families on nonexpansive mappings," Pacific Journal of Mathematics, vol. 53, no. 2, pp. 487-493, 1974.

[7] R. DeMarr, "Common fixed points for commuting contraction mappings," Pacific Journal of Mathematics, vol. 13, no. 4, pp. 1139-1141, 1963.

[8] K.-K. Tan and H.-K. Xu, "An ergodic theorem for nonlinear semigroups of lipschitzian mappings in banach spaces," Nonlinear Analysis: Theory, Methods \& Applications, vol. 19, no. 9, pp. 805-813, 1992.

[9] S. Fuan, R. Ullah, G. Rahmat, M. Numan, S. I. Butt, and X. Ge, "Approximate fixed point sequences of an evolution family on a metric space," Journal of Mathematics, vol. 2020, Article ID 1647193, 6 pages, 2020.

[10] A. A. Abdou and M. A. Khamsi, "On common fixed points in modular vector spaces," Fixed Point Theory and Applications, vol. 229, no. 1, 2015.

[11] M. Bachar, B. A. Bin Dehaish, and M. A. Khamsi, "Approximate fixed points," Fixed Point Theory and Graph Theory, pp. 99-138, 2016.

[12] A. Buthinah, B. Dehaish, and M. A. Khamsi, "Approximation common fixed points of semigroups in metric spaces," Fixed Point Theory and Applications, vol. 51, 2015.

[13] M. A. Khamsi and W. M. Kozlowski, "On asymptotic pointwise contractions in modular function spaces," Nonlinear Analysis: Theory, Methods \& Applications, vol. 73, no. 9, pp. 2957-2967, 2010. 
[14] M. A. Khamsi and W. M. Kozlowski, "On asymptotic pointwise nonexpansive mappings in modular function spaces," Journal of Mathematical Analysis and Applications, vol. 380, no. 2, pp. 697-708, 2011.

[15] W. A. Kirk and H.-K. Xu, "Asymptotic pointwise contractions," Nonlinear Analysis: Theory, Methods \& Applications, vol. 69, no. 12, pp. 4706-4712, 2008.

[16] N. Hussain and M. A. Khamsi, "On asymptotic pointwise contractions in metric spaces," Nonlinear Analysis: Theory, Methods \& Applications, vol. 71, no. 10, pp. 4423-4429, 2009.

[17] M. A. Khamsi, "Nonlinear semigroups in modular function spaces," Japanese Journal of Mathematics, vol. 379, pp. 1-9, 1992.

[18] K. Menger, "Untersuchungen über allgemeine metrik," Mathematische Annalen, vol. 100, no. 1, pp. 75-163, 1928.

[19] S. Reich and I. Shafrir, "Nonexpansive iterations in hyperbolic spaces," Nonlinear Analysis: Theory, Methods \& Applications, vol. 15, no. 6, pp. 537-558, 1990.

[20] J. A. Clarkson, "Uniformly convex spaces," Transactions of the American Mathematical Society, vol. 40, no. 3, p. 396, 1936.

[21] K. Goebel, T. Sekowski, and A. Stachura, "Uniform convexity of the hyperbolic metric and fixed points of holomorphic mappings in the Hilbert ball," Nonlinear Analysis: Theory, Methods \& Applications, vol. 4, no. 5, pp. 1011-1021, 1980.

[22] K. Goebel and S. Reich, Uniform Convexity, Hyperbolic Geometry, And Nonexpansive Mappings, Marcel Dekker Inc., New York, NY, USA, 1984.

[23] M. A. Khamsi and A. R. Khan, "Inequalities in metric spaces with applications," Nonlinear Analysis: Theory, Methods \& Applications, vol. 74, no. 12, pp. 4036-4045, 2011.

[24] M. A. Khamsi, "On metric spaces with uniform normal structure," Proceedings of the American Mathematical Society, vol. 106, no. 3, p. 723, 1989.

[25] I. Stewart and T. David, Algebraic Number Theory and Fermat's Last Theorem, CRC Press, Boco Raton, FL, USA, 2001.

[26] T. Suzuki, "The set of common fixed points of a one-parameter continuous semigroup of mappings is $F(T(1)) \cap F(T(\sqrt{(2)})$," Proceedings of the American Mathematical Society, vol. 134, no. 3, pp. 673-681, 2006.

[27] T. Kuczumow, "An almost convergence and its applications," Annales Universitatis Mariae Curie-Sklodowska, vol. 32, pp. 79-88, 1978.

[28] H. Fukhar-ud-din and M. A. Khamsi, "Approximating common fixed points in hyperbolic spaces," Fixed Point Theory and Applications, vol. 113, 2014.

[29] D. Göhde, "Zum prinzip der kontraktiven abbildung," Mathematische Nachrichten, vol. 30, no. 3-4, pp. 251-258, 1965.

[30] G. Rahmat, S. Hussain, and S. I. Butt, "Strong convergence to a fixed point of a subfamily of a non-expansive evolution family in Hilbert spaces," 2021.

[31] G. Rahmat, T. Shah, M. Sarwar, H. Aydi and H. Alsamir, "Common fixed points of a subfamily of a non-expansive periodic evolution family on strictly convex Banach space," 2021.

[32] M. Shah, G. Rahmat, and S. I. A. Shah, "Iterative processes of convergence to a fixed point of an evolution family on a Banach spaces," 2020. 